\title{
Combined radiofrequency and chemoembolization vs. chemoembolization in management of hepatocellular
} carcinoma

\author{
Ahmed Kamal El Dorry', Eman Mahmoud Fathy Barakat ${ }^{2}$, Amal Tohamy Abd ElMoez ${ }^{2}$, \\ Mervat Abd Fatah Mawad ${ }^{3}$, Nevien Fouad El-Fouly ${ }^{3}$, Mohamed Kamal Shaker ${ }^{2}$
}

${ }^{1}$ Department of Radiology Medicine, Faculty of Medicine, Ain Shams University, Cairo 11566, Egypt

${ }^{2}$ Department of Tropical Medicine, Faculty of Medicine, Ain Shams University, Cairo 11566, Egypt

${ }^{3}$ Atomic Energy Authority, Cairo 13759, Egypt

\begin{abstract}
Aim: Hepatocellular carcinoma ( $\mathrm{HCC})$ is one of the most common cancers in the world. If left untreated, liver cancer has a poor prognosis with more than $90 \%$ of patients dying of the disease within 5 years of diagnosis. The aim of this study is to assess the value of combined radiofrequency ablation (RFA), followed by trans-arterial chemoembolization (TACE) in the management of HCC. Methods: Fifty HCC patients with chronic liver disease were categorized into two groups according to the modality of locoregional treatment: $25 \mathrm{HCC}$ patients treated with RFA followed by TACE within 5 days and 25 HCC patients treated with TACE only. Results: Complete response was achieved in $100 \%$ and $84 \%$ of the HCC patients after 1 month from combined RFA-TACE therapy and TACE only respectively. The rate of objective response after 7 months was $84 \%$ and $44 \%$ in the RFA-TACE and TACE groups respectively. One year disease free survival rate was $56 \%$ and $24 \%$ in RFA-TACE and TACE groups respectively, and overall survival rate was $88 \%$ in the RFA-TACE group and $80 \%$ in the TACE only group. Conclusion: Combined RFA-TACE appears to be an effective modality and superior to TACE only for the treatment of HCC.
\end{abstract}

Key words: Hepatocellular carcinoma; radiofrequency; trans-arterial chemoembolization

Address for correspondence:

Dr. Amal Tohamy Abd ElMoez, Department of Tropical Medicine, Faculty of Medicine, Ain Shams University, Cairo 11566, Egypt. E-mail: d_amal_tohamy@yahoo.com

Received: 27-12-2014, Accepted: 23-02-2015

\section{INTRODUCTION}

Hepatocellular carcinoma (HCC) is the fifth most common cancer in the world, with over 600,000 new diagnoses/year. ${ }^{[1]}$ In Egypt, the annual incidence of HCC showed a significant rising trend from $4.0 \%$ in 1993 to $7.2 \%$ in 2002. ${ }^{[2]}$ Radiofrequency ablation (RFA) is a locoregional modality for tumor ablation which often allows for greater preservation

\begin{tabular}{|l|c|}
\hline \multicolumn{2}{|c|}{ Access this article online } \\
\hline \multirow{2}{*}{ Website: } & Quick Response Code \\
http://www.hrjournal.net/ & \\
\hline & DOI: \\
10.4103/2394-5079.154355 & \\
&
\end{tabular}

of unaffected hepatic parenchyma. ${ }^{|3|}$ The greatest success rate was achieved in non-infiltrating tumors of sizes 3-5 cm..$^{[4]}$ Trans-arterial chemoembolization (TACE) is an intra-arterial infusion of chemotherapy, which blocks (embolizes) small blood vessels, depriving the tumor of its needed blood supply. However, this procedure is not curative. ${ }^{[5]}$ The combination of RFA and TACE induces coagulative necrosis in large areas without any possibility of revascularization. ${ }^{[6]}$

The aim of this study was to assess the efficacy of combined RFA followed by chemoembolization in the management of HCC.

\section{METHODS}

This cross-sectional randomized controlled prospective study was performed at the HCC Clinic at Ain Shams University. 


\section{Ethical consideration}

Ethical approval was obtained from the Local Ethical Committee of the Department of Tropical Medicine, Ain Shams University. Written informed consent was obtained from all participants. The right to refuse participation was emphasized.

\section{Patients}

The study included 50 cases of diagnosed HCC on top of chronic liver disease divided into two groups according to treatment modality. Group 1: 25 HCC patients underwent TACE only as a control group with $10(40 \%)$ patients with single tumor sizes of $5 \mathrm{~cm}$ and $15(60 \%)$ patients with single tumor sizes of 5-7 cm. Group 2: 25 HCC patients underwent RFA followed by TACE (RFA-TACE) within 5 days with 14 (56\%) patients with single tumor sizes of $5 \mathrm{~cm}$ and $11(44 \%)$ patients with single tumor sizes of $5-7 \mathrm{~cm}$.

All patients fit clinical, biochemical, radiological (ultrasound) criteria of chronic liver disease and had positive hepatitis $\mathrm{C}$ virus (HCV) Ab and/or HbsAg. HCC was diagnosed by evidence of high $\alpha$ fetoprotein (AFP) (> $200 \mathrm{ng} / \mathrm{mL}$ ) and/or presentation of HCC characteristics by triphasic spiral abdominal computed tomography (CT), according to American Association for the Study of Liver Diseases guidelines. ${ }^{[7]}$

\section{Inclusion criteria}

We included patients with Child-Pugh class A or B presentations, prothrombin concentrations of $>50 \%$, platelet counts of $>50,000$ per $\mathrm{mm}^{3}$, and ultrasound detection of the lesion to be allowed for percutaneous loco-regional ablation therapy.

\section{Exclusion criteria}

Patients with Child-Pugh class C presentations tumors in inaccessible sites or close to vital structures, malignant main portal vein involvement and/or extrahepatic metastases. All patients were categorized according to the Barcelona-Clinic liver cancer (BCLC) Staging System of HCC pre- and post-treatment to evaluate the general condition, performance status of liver functionality, Child-Pugh scores and Okuda stage..$^{[8-11]}$

\section{Procedures}

All of the enrolled patients were subjected to tests of: complete blood count (CBC), alanine transaminase, aspartate transaminase, serum albumin, serum bilirubin, prothrombin time and international normalized ratio (INR), hepatitis markers (HCV Ab, HBsAg and HBcAb), AFP, chest X-ray, abdominal ultrasonography and abdominal triphasic spiral CT.

In post-treatment follow-up studies, all patients were subjected to the following evaluation measures 1-month afterwards and then every 3 months following the procedure: full clinical examination, $\mathrm{CBC}$, prothrombin concentration, liver function tests, kidney function, AFP and spiral CT of abdomen. Classification of response was conducted according to the European Association for the Study of Liver amendments that take into account the reduction in viable tumor volume due to TACE-induced necrosis as follows. Complete response (CR): complete disappearance of all known disease and no new lesions; partial response (PR): at least $50 \%$ reduction in total tumor load of all measurable lesions; progressive disease (PD): at least 25\% increase in size of one or more measurable lesions or the appearance of new lesions; stable disease (SD): does not qualify for CR/ PR or PD. ${ }^{[7]}$

Radiofrequency ablation was performed percutaneously under ultrasound guidance after general anesthesia. RITA (Mountain View, California, USA) and Boston Scientific (Natick, Massachusetts, USA) expandable-type electrodes were used. These electrodes had multiple thin curved monopolar electrodes extending from the central cannula (18-14 gauge). Radiofrequency emanates from each of these hooks, resulting in increased coagulation. Also Valley Lab (Boulder, Colorado, USA) cooled-tip electrodes (17 gauge) were used. These electrodes have two hollow lumens that permit continuous internal cooling of the tip. Using percutaneous endovascular techniques, TACE was performed by selective catheterization of the hepatic segmental arteries nourishing the lesions, using either 5-F catheters (Simmons 1 and Cobra; Mallinckrodt, St. Louis, USA or Hydrophilic Simmons 1 and Cobra; Terumo, Tokyo, Japan) or 3-F coaxial microcatheters (Tracker 18; Vascular Access System, Target, St. José, USA; SP Catheter; Terumo). TACE was used to deliver potent anticancer drugs directly into tumor-feeding arteries. As a result, tumors were exposed to very high drug concentrations, while systemic exposure was minimized. The cytotoxic lipiodol mixture was prepared by mixing $100 \mathrm{mg}$ adriablastin powder with $10 \mathrm{~mL}$ of saline, water soluble contrast and $10 \mathrm{~mL}$ of oily contrast (Lipiodol Ultra-Fluid; Jubilant HollisterStier General Partnership 16751 Trans-Canada Highway, Kirkland, Quebec, Canada) to ensure a homogenous mixture. Embolization was done by mixing small pieces of gel foam particles and water soluble contrast.

\section{Statistical analysis}

The collected data were statistically analyzed using the program SPSS (Statistical Package for Social Sciences, software version 18.0, Echosoft Corporation, USA). Data were expressed as mean \pm standard deviation for quantitative parametric measures in addition to median percentiles for quantitative non-parametric measures and both number and percentage for categorized data. The following tests were 
used: Student's $t$-test, Wilcoxon rank sum test, Chi-square, and Kaplan-Meier.

\section{RESULTS}

Both groups were matched with regards to age and sex. Comparison between both groups regarding the different scoring systems shows no statistically significant difference $(P>0.05)$. There were $23(92 \%)$ Child-Pugh class A and $2(8 \%)$ class B patients in the TACE only group vs. 24 (96\%) and $1(4 \%)$ in the RFA-TACE group. Performance status grades of 0 were present in $18(72 \%)$ patients and $7(28 \%)$ with grade 0 in TACE vs. 14 (56\%) and 11 (44\%) in RFA-TACE. BCLC stage B was found in $18(72 \%)$ and $7(28 \%)$ in stage C TACE vs. 14 (56\%) and 11 (44\%) in RFA-TACE respectively [Table 1]. Table 2 shows the response to the treatment after 1 month, with all patients underwent RFA-TACE achieved CR, but with no statistically significant difference between both groups $(P>0.05)$. After 7 months of treatment, the rate of the objective response (which includes both $C R$ and $P R$ for at least 6 months) was higher in RFA-TACE than that of TACE alone $(P<0.01)$. It was noted that lesions of more than $5 \mathrm{~cm}$ were more liable to PR and PD after chemoembolization alone. Performance status was improved in the RFA-TACE groups, shifting from a grade of 1-0 $(P<0.01)$. As summarized in Table 3, 1-year total recurrence rates and local tumor progression rates were higher in patients that underwent chemoembolization alone $(P<0.01)$. In Table 4, 1-year disease free survival rates and overall survival rates were higher after the combined therapy $(P<0.001)$. Figure 1 shows the median survival time for the two studied groups, which was 13 months with no statistically significant difference $(P>0.05)$. Comparison between both groups with regards to survival rates at 7 months and at 1 year is shown in Figure 2. No major complication was reported after combined therapy or after TACE only; only post-embolization syndrome was reported as a minor complication in $68 \%$ and $72 \%$ of the patients in RFA-TACE and TACE groups, respectively.

\section{DISCUSSION}

Hepatocellular carcinoma is the fifth most common cancer worldwide and the third leading cause of cancer-related mortality, ${ }^{[12]}$ with its incidence increasing worldwide ranging between 3\% and 9\% annually. ${ }^{[3]}$ The European Association for the Study of Liver and the American Association for the Study of Liver Diseases recommends RFA as a non-surgical technique for the treatment of early stage HCC (Child-Pugh class A or B, solitary HCCs or up to 3 nodules with each $\leq 3 \mathrm{~cm}$ in size). ${ }^{[7]}$ TACE has become the treatment of choice
Table 1: Scoring systems before treatment

\begin{tabular}{lccc}
\hline Variable & \multicolumn{2}{c}{$n=25(n(\%))$} & $P$ \\
\cline { 2 - 3 } & TACE & RFA-TACE & \\
\hline Child-Pugh class & $23(92.0)$ & $24(96.0)$ & $>0.05$ \\
B & $2(8.0)$ & $1(4.0)$ & \\
Okuda stage & & & \\
I & $24(96.0)$ & $25(100)$ & \\
II & $1(4.0)$ & $0(0)$ & \\
PST & & & \\
0 & $18(72.0)$ & $14(56.0)$ & \\
1 & $7(28.0)$ & $11(44.0)$ & \\
BCLC & $18(72.0)$ & $14(56.0)$ & $>0.05$ \\
B & $7(28.0)$ & $11(44.0)$ & \\
C & RFA: radiofrequency ablation; TACE: trans-arterial chemoembolization; \\
PST: performance status test; BCLC: Barcelona-Clinic liver cancer &
\end{tabular}

Table 2: Treatment response in the studied groups

\begin{tabular}{|c|c|c|c|}
\hline & \multicolumn{2}{|c|}{$n=25(n(\%))$} & \multirow[t]{2}{*}{$P$} \\
\hline & TACE & RFA-TACE & \\
\hline \multicolumn{4}{|l|}{ After 1 month } \\
\hline Complete response & $21(84)$ & $25(100)$ & $>0.05$ \\
\hline Partial response & $2(8)$ & $0(0)$ & \\
\hline Progressive disease & $2(8.0)$ & $0(0)$ & \\
\hline \multicolumn{4}{|l|}{ After 7 months } \\
\hline Objective response & $11(44)$ & $21(84)$ & $<0.01$ \\
\hline
\end{tabular}

Table 3: Overall recurrence rates at 13 months following the procedure

\begin{tabular}{lccc}
\hline & \multicolumn{2}{c}{$n=25(n(\%))$} & $P$ \\
\cline { 2 - 3 } & TACE & RFA-TACE & \\
\hline $\begin{array}{l}\text { Total recurrence of HCC } \\
\text { (same lesion and/or new lesion) }\end{array}$ & $18(72)$ & $9(36)$ & $<0.05$ \\
$\begin{array}{l}\text { Recurrence of HCC in same lesion only } \\
\text { RFA: radiofrequency ablation; TACE: trans-arterial chemoembolization; }\end{array}$ & \\
HCC: hepatocellular carcinoma & & & \\
HC (16) & $<0.01$ \\
\hline
\end{tabular}

Table 4: The disease free survival rate and the survival rate in the studied groups

\begin{tabular}{lccc}
\hline & \multicolumn{2}{c}{$n=25(n(\%))$} & $P$ \\
\cline { 2 - 3 } & TACE & RFA-TACE & \\
\hline Disease free survival rate at 1 year & $6(24)$ & $14(56)$ & $<$ \\
Overall survival rate at 1 year & $20(80)$ & $22(88)$ & $>0.001$ \\
\hline RFA: radiofrequency ablation; TACE: trans-arterial chemoembolization &
\end{tabular}

for multinodular HCC, ${ }^{[14]}$ and for large HCCs in patients who are not surgical candidates. ${ }^{[15]}$ Another promising role of RFA is to be combined with TACE for the treatment of intermediate and large tumors, ${ }^{[16]}$ so as to obtain a large area of coagulation. ${ }^{[17]}$ The combination of TACE with RFA has two theoretical merits: (1) occlusion of hepatic arterial flow by means of embolization may contribute to the decrease in the heat-sink effects during RFA and increase the ablation volume by RFA; and (2) combined treatment may have the 


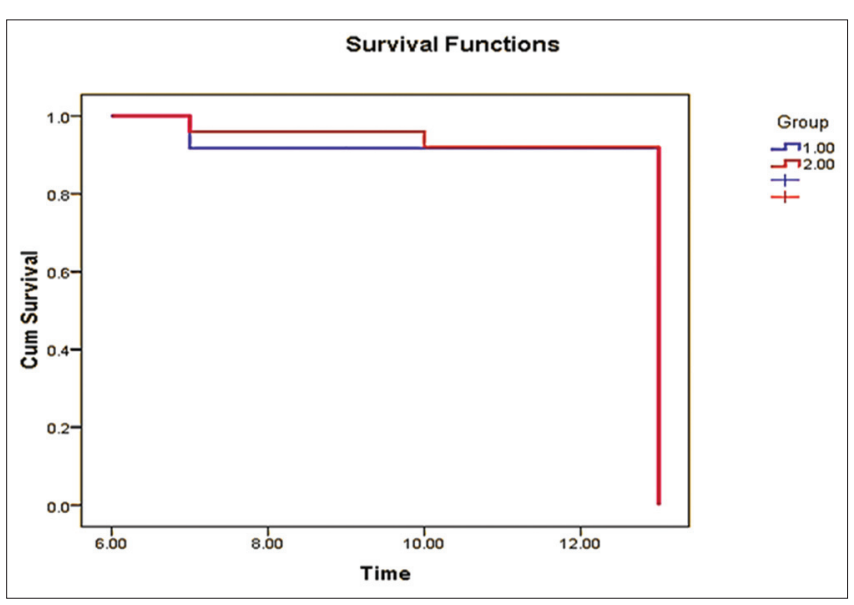

Figure 1: Kaplan-Meier curve showing the median survival time for the two groups. Kaplan-Meier curve showed the median survival time for the two groups, which were 13 months and there was no statistically significant difference

effect of anticancer agents on cancer cells, which is enhanced by the hyperthermia. ${ }^{[18]}$ In this work we evaluated the efficacy of combined RFA followed by TACE (RFA-TACE) in single HCCs $(5-7 \mathrm{~cm})$ in comparison to TACE only. The study results showed CR after 1 month in $100 \%$ and $84 \%$ of patients in combined RFA-TACE and TACE alone groups respectively. This is in agreement with other groups of researchers who reported that CR was achieved in $79.9 \%$ and $92.5 \%$ of HCC patients after combined (TACE-RFA) and RFA therapy respectively. ${ }^{[19,20]}$ In this work, both medium HCCs $(5 \mathrm{~cm})$ and large HCCs $(>5 \mathrm{~cm})$ achieved CR in $100 \%$ of patients in the RFA-TACE group, whereas Wang et al. ${ }^{[21]}$ showed that CR was achieved in $57.6 \%$ of medium sized HCCs and in $6 \%$ of large sized HCCs after (TACE-RFA) therapy. Another study documented that $C R$ was achieved in $40 \%$ of patients with large sized HCC after (TACE-RFA) therapy. ${ }^{[22]}$ In our study, the rate of objective responses after 7 months (CR and/or PR for at least 6 months) were $84 \%$ and 44\% in RFA-TACE and TACE groups respectively, while Cheng et al. ${ }^{[23]}$ reported that the rate of objective response after 6 months was 54\% and 35\% in TACE-RFA and TACE group respectively. In the current work, there was improvement in the performance status of many patients following RFA-TACE therapy as $44 \%$ of patients shifted from a PST score of 1-0 after 1 month of the combined therapy while $4 \%$ of patients were shifted from a PST of 0-1 after TACE only. Sacco et al. ${ }^{[24]}$ revealed that there was worsening of clinical status in patients after TACE only. These results ensured that combined therapy had a positive impact on the performance status of HCC patients, also improved their survival and disease free survival rate and shifted them from BCLC stage $\mathrm{C}$, where patients were restricted to certain supportive medical treatments, to stage $\mathrm{B}$, where other different modalities could be implemented for HCC patients and with a good prognosis. In this study, local tumor progression rate at 1 year was $16 \%$ after RFA-TACE

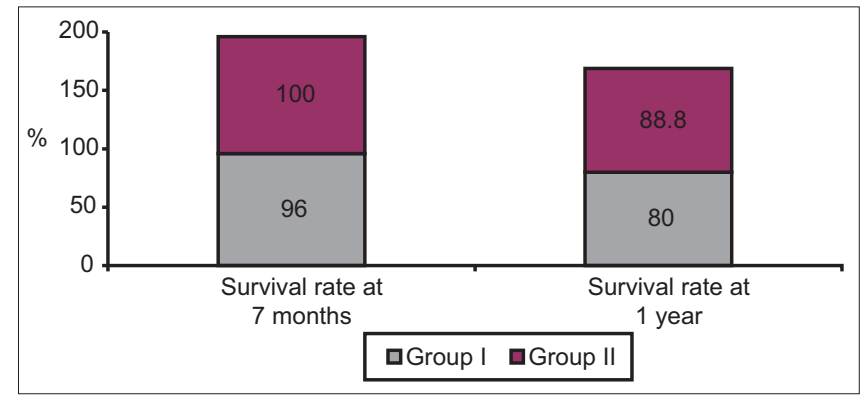

Figure 2: Survival rates at 7 months and 1 year. Survival rate at 7 months was $100 \%$ in radiofrequency ablation (RFA) + trans-arterial chemoembolization (TACE) group and $96 \%$ in TACE group. One year survival rate was $88.8 \%$ in RFA + TACE group and $80 \%$ in TACE group with statistically significant difference. RFA: radiofrequency ablation; TACE: trans-arterial chemoembolization

therapy. This result is comparable to Takaki et al..$^{[22]}$ and Kim et al. ${ }^{[25]}$ who reported that local tumor progression rate at 1 year was $15 \%$ and $9 \%$ after TACE-RFA respectively. In the present work, no major complications were reported after combined therapy or after TACE only. Only post-embolization syndrome was reported as a minor complication in $68 \%$ and $72 \%$ in RFA-TACE and TACE groups respectively. Another study reported that major complications were observed in $2.2 \%$ of patients and minor complications were observed in $2.2 \%$ of patients post-combined TACE-RFA therapy. ${ }^{[19]}$ Takaki et al. ${ }^{[22]}$ stated that a minor complication was observed in $3 \%$ of patients post-combined TACE-RFA therapy. In the current study, 1-year survival rate was $88 \%$ and $80 \%$ in RFA-TACE and TACE only respectively, which is comparable with previous studies showing 1-year survival rates at $93 \%, 98 \%$ and $100 \%$ in TACE-RFA treated groups. ${ }^{\mid 19,23,26]}$ The present study found that, 1 -year recurrence-free survival rate was $56 \%$ and $24 \%$ in RFA-TACE and TACE respectively, which is in concordance with other studies reporting 1-year recurrence free survival rates of $74 \%$ and $64.5 \%{ }^{[22,26]}$ Comparative studies have previously described TACE-RFA, a combined technique in which TACE was performed before RFA, and proved that it is much more better than mono-therapy, especially in medium and large sized HCC lesions. Although it is different from the technique discussed in this study in which TACE was done after RFA (RFA-TACE), both have nearly the same results, with RFA-TACE presenting better responses with regards to CR and objective response than TACE-RFA and mono-therapy, especially in management of medium sized HCC. In TACE-RFA, occlusion of hepatic arterial flow by means of embolization may contribute to the decrease in the heat-sink effects during RFA, increase in the ablation volume, and induce coagulation necrosis in large areas without any possibility of revascularization. During RFA, the high rate of local recurrence may be due to residual cancer cells or adjacent microscopic satellite tumor nodules, so TACE could be used as an adjuvant therapy after RFA to eradicate the peripheral viable tissue and micro-metastasis with more concentrated 
local chemotherapeutic agents, also enhancing the effects of anticancer agents on cancer cells by the hyperthermia following RFA. So our conclusion is combined RFA and TACE appear to be effective modality and superior to TACE alone for the treatment of HCC

\section{REFERENCES}

1. Parkin DM, Bray F, Ferlay J, Pisani P. Global cancer statistics, 2002. CA Cancer J Clin 2005;55:74-108.

2. el-Zayadi AR, Badran HM, Barakat EM, Attia Mel-D, Shawky S, Mohamed MK, Selim O, Saeid A. Hepatocellular carcinoma in Egypt: a single center study over a decade. World J Gastroenterol 2005;11:5193-8.

3. McGhana JP, Dodd GD 3rd. Radiofrequency ablation of the liver: current status. AJR Am J Roentgenol 2001;176:3-16.

4. Livraghi T, Goldberg SN, Lazzaroni S, Meloni F, Ierace T, Solbiati L, Gazelle GS. Hepatocellular carcinoma: radio-frequency ablation of medium and large lesions. Radiology 2000;214:761-8.

5. Janis and Friends, Hepatitis C Support web site, Liver Cancer; 2008. Available from: http://www.janisandfriendshepatitiscsupport.com. [Last accessed on 2012 Oct 1].

6. Kurokohchi K, Watanabe S, Masaki T, Hosomi N, Funaki T, Arima K, Yoshida S, Miyauchi Y, Kuriyama S. Combined use of percutaneous ethanol injection and radiofrequency ablation for the effective treatment of hepatocelluar carcinoma. Int J Oncol 2002;21:841-6.

7. Bruix J, Sherman M. Practice Guidelines Committee, American Association for the Study of Liver Diseases. Management of hepatocellular carcinoma. Hepatology 2005;42:1208-36.

8. Llovet JM, Burroughs A, Bruix J. Hepatocellular carcinoma. Lancet 2003;362:1907-17.

9. Mallick I. What is the performance status? Health Disease and Condition Content is reviewed by the Medical Review Board; 2007. Available from: http://www.About.com. [Last accessed on 2012 Oct 1].

10. Mor E, Tur-Kaspa R, Sheiner P, Schwartz M. Treatment of hepatocellular carcinoma associated with cirrhosis in the era of liver transplantation. Ann Intern Med 1998;129:643-53.

11. Okuda K, Ohtsuki T, Obata H, Tomimatsu M, Okazaki N, Hasegawa H, Nakajima Y, Ohnishi K. Natural history of hepatocellular carcinoma and prognosis in relation to treatment. Study of 850 patients. Cancer 1985;56:918-28.

12. Al Knawy B, Reddy KR, Bolondi L, editors. Preface. In: Hepatocellular Carcinoma: a practical approach. United Kingdom: Informa Healthcare; 2009.

13. Velázquez RF, Rodríguez M, Navascués CA, Linares A, Pérez R, Sotorríos NG, Martínez I, Rodrigo L. Prospective analysis of risk factors for hepatocellular carcinoma in patients with liver cirrhosis. Hepatology 2003;37:520-7.

14. Llovet JM, Bruix J. Systematic review of randomized trials for unresectable hepatocellular carcinoma: chemoembolization improves survival. Hepatology 2003;37:429-42.

15. Miraglia R, Pietrosi G, Maruzzelli L, Petridis I, Caruso S, Marrone G, Mamone G, Vizzini G, Luca A, Gridelli B. Efficacy of transcatheter embolization/chemoembolization (TAE/TACE) for the treatment of single hepatocellular carcinoma. World J Gastroenterol 2007;13:2952-5.

16. N'Kontchou G, Mahamoudi A, Aout M, Ganne-Carrié N, Grando V, Coderc E, Vicaut E, Trinchet JC, Sellier N, Beaugrand M, Seror O. Radiofrequency ablation of hepatocellular carcinoma: long-term results and prognostic factors in 235 Western patients with cirrhosis. Hepatology 2009;50:1475-83.

17. Takaki H, Yamakado K, Nakatsuka A, Fuke H, Murata K, Shiraki K, Takeda K. Radiofrequency ablation combined with chemoembolization for the treatment of hepatocellular carcinomas $5 \mathrm{~cm}$ or smaller: risk factors for local tumor progression. J Vasc Interv Radiol 2007;18:856-61.

18. Rhim H, Lim HK, Choi D. Current status of radiofrequency ablation of hepatocellular carcinoma. World J Gastrointest Surg 2010;2:128-36.

19. Yamakado K, Nakatsuka A, Takaki H, Yokoi H, Usui M, Sakurai H, Isaji S, Shiraki K, Fuke H, Uemoto S, Takeda K. Early-stage hepatocellular carcinoma: radiofrequency ablation combined with chemoembolization versus hepatectomy. Radiology 2008;247:260-6.

20. Peng ZW, Chen MS, Liang HH, Gao HJ, Zhang YJ, Li JQ, Zhang YQ, Lau WY. A case-control study comparing percutaneous radiofrequency ablation alone or combined with transcatheter arterial chemoembolization for hepatocellular carcinoma. Eur J Surg Oncol 2010;36:257-63.

21. Wang YH, Liu JF, Li F, Li A, Liu Q, Liu DB, Liu DG, Wang YJ. Radiofrequency ablation combined with transarterial chemoembolization for unresectable primary liver cancer. Chin Med J (Engl) 2009;122:889-94.

22. Takaki H, Yamakado K, Uraki J, Nakatsuka A, Fuke H, Yamamoto N, Shiraki K, Yamada T, Takeda K. Radiofrequency ablation combined with chemoembolization for the treatment of hepatocellular carcinomas larger than $5 \mathrm{~cm}$. J Vasc Interv Radiol 2009;20:217-24.

23. Cheng BQ, Jia CQ, Liu CT, Fan W, Wang QL, Zhang ZL, Yi CH. Chemoembolization combined with radiofrequency ablation for patients with hepatocellular carcinoma larger than $3 \mathrm{~cm}$ : a randomized controlled trial. JAMA 2008;299:1669-77.

24. Sacco R, Bertini M, Petruzzi P, Bertoni M, Bargellini I, Bresci G, Federici G, Gambardella L, Metrangolo S, Parisi G, Romano A, Scaramuzzino A, Tumino E, Silvestri A, Altomare E, Vignali C, Capria A. Clinical impact of selective transarterial chemoembolization on hepatocellular carcinoma: a cohort study. World J Gastroenterol 2009;15:1843-8.

25. Kim JH, Won HJ, Shin YM, Kim SH, Yoon HK, Sung KB, Kim PN. Medium-sized (3.1-5.0 cm) hepatocellular carcinoma: transarterial chemoembolization plus radiofrequency ablation versus radiofrequency ablation alone. Ann Surg Oncol 2011;18:1624-9.

26. Kagawa T, Koizumi J, Kojima S, Nagata N, Numata M, Watanabe N, Watanabe T, Mine T; Tokai RFA Study Group. Transcatheter arterial chemoembolization plus radiofrequency ablation therapy for early stage hepatocellular carcinoma: comparison with surgical resection. Cancer 2010;116:3638-44.

How to cite this article: El Dorry AK, Fathy Barakat EM, Abd EIMoez AT, Fatah Mawad MA, El-Fouly NF, Shaker MK. Combined radiofrequency and chemoembolization vs. chemoembolization in management of hepatocellular carcinoma. Hepatoma Res 2015;1:19-23.

Source of Support: Nil. Conflict of Interest: None declared. 